\title{
Dynamical Cosmological Constant From A Very Recent Phase Transition
}

\author{
JOHN MCDONALD ${ }^{1}$ \\ Department of Physics and Astronomy, University of Glasgow, Glasgow G12 8QQ, SCOTLAND
}

February 5, 2020

\begin{abstract}
Observation indicates that the expansion of the Universe is accelerating and favours a dynamical cosmological constant, $\Lambda(t)$. We consider the possibility that this is due to a scalar field which has undergone a very recent phase transition. We study a simple class of model, corresponding to a $\phi^{4}$ potential with a time-dependent mass squared term. For the models considered the phase transition occurs at a red shift $z \leq 1.2$. The evolution of the equation of state $\omega_{\phi}$ and energy density $\rho_{\phi}$ with time is distinct from existing dynamical $\Lambda$ models based on slowly rolling fields, with $\omega_{\phi}$ and $\rho_{\phi}$ rapidly changing in a characteristic way following the transition. The $\phi$ energy density is composed of a time-dependent vacuum energy and coherently oscillating condensate component with a negative pressure. The condensate component will typically collapse to form nontopological soliton lumps, ' $\phi$-axitons', which smoothly populate the Universe.
\end{abstract}

\footnotetext{
${ }^{1}$ mcdonald@physics.gla.ac.uk
} 
Perhaps the most remarkable cosmological observation of recent times is the accelerating expansion of the Universe [1]. This requires the existence of an energy density which has a negative pressure and which is smooth on the $10 \mathrm{Mpc}$ scales relevant to dynamical estimates of the density of conventional dark matter. Other evidence for this comes from estimates of the age of the Universe, the Hubble constant, the baryon fraction in clusters, the galactic power spectrum and CMB measurements [2, 3]. The simplest explaination for a smooth energy density with a negative pressure is a timeindependent cosmological constant, $\Lambda$. However, there are a number of problems with a fixed cosmological constant. The first is that it is difficult to understand why the cosmological constant should just be dominating the energy density at the present epoch | 1 . This has led to two different views. One is that the cosmological constant is due to the evolution of a scalar field ('tracking solution' [5]) whose energy density becomes significant recently due to dynamical effects. In general this approach has difficulties with nucleosynthesis and the present equation of state [6]. (A particularly promising version which may overcome these problems is given in [7].) The other view is that the cosmological constant has become dominant in the present epoch due to anthropic selection (AS) [4]. However, even if we assume that AS is responsible for the dominance of the cosmological constant, there are still problems. It is difficult to understand why a fixed $\Lambda$ is so small compared to the mass scales of particle physics $\left(\Lambda \sim 10^{-120} \mathrm{M}_{\mathrm{Pl}}^{4}\right)$. This has led to the suggestion that the absolute minimum of the vacuum energy should be exactly zero and that the smooth energy density is due to the evolution of a scalar field towards the minimum, resulting in a dynamical cosmological constant, $\Lambda(t)[8,9,10,12,13]$. There is also observational evidence in support of a specifically dynamical cosmological constant [11]. The amplitude of the COBE-normalized galaxy clustering power spectrum is too large in the case of a fixed $\Lambda$. However, if the effective cosmological constant is decreasing with time, then the amplitude of the power spectrum is reduced, alleviating the problem [11].

There have been a number of suggestions regarding the nature of the dynamical cosmological constant. Most popular are models based on pseudo-Nambu-Goldstone bosons (PNGBs) [10, 8, 9, 12], exponential potentials [5, 6, 8, 12] and inverse-power 
law potentials [13, 8, 9]. All of these produce characteristic time-dependent energy densities $\rho_{\phi}$ and pressures $p_{\phi}\left(\equiv \omega_{\phi} \rho_{\phi}\right)$ which come to dominate at recent red-shifts. For the PNGB models, the pressure slowly tends from negative values towards zero. For tracking models based on exponential and inverse-power law potentials, on the other hand, the pressure is evolving from a value typically positive and close to zero (corresponding to matter tracking) towards a negative value. This should allow the models to be distinguished by precision CMB angular spectra measurements [12]. All of these models are based on very light scalar fields $\left(m_{\phi} \lesssim H_{o}\right.$, where $H_{o}$ is the present value of the expansion rate) which are slowly rolling at the present time, $|\dot{\phi} / \phi| \lesssim H_{o}$.

In the present paper we wish to introduce an alternative model for a dynamical cosmological constant. This is based on the idea that the negative pressure energy density is associated with a conventional metastable false vacuum. This is perhaps the simplest form of negative pressure energy density in particle physics models. However, in order to have a dynamical cosmological constant we require that a phase transition from the metastable phase has occured recently. To achieve this we will consider the scalar field $\phi$ to have a time dependent mass squared term which has recently become negative. We will refer to this scheme as the 'very recent phase transition' (VRPT) scenario for a dynamical cosmological constant. The VRPT scenario is quite distinct from previous models based on slowly rolling scalar fields. In particular, there will be no need for the mass scale of the scalar to be extremely small, and the pressure and energy density will be rapidly evolving in a characteristic way at recent times following the phase transition. This should allow the VRPT scenario to be distinguished from the others by precision CMB measurements.

We will consider the usual spontaneous symmetry breaking potential for a real scalar field with $\phi \leftrightarrow-\phi$ symmetry门,

$$
V(\phi)=-\frac{\mu^{2}(t)}{2} \phi^{2}+\frac{\lambda}{4} \phi^{4}+\Lambda \quad ; \quad \Lambda=\frac{\mu_{o}^{4}}{4 \lambda}
$$

\footnotetext{
${ }^{1}$ This can be generalized to a complex scalar. For a real scalar field domain walls may form during the VRPT, whilst for a complex field global strings may form.
} 
where

$$
\mu^{2}(t)=\mu_{o}^{2}\left(1-\left(\frac{a_{c}}{a}\right)^{n}\right)
$$

and $a_{c}$ is the scale factor at the time of the transition. In general the VRPT scenario requires an additional time-dependent $\phi^{2}$ term, which we will refer to as a 'stabilizing interaction'. We will discuss some possible sources for the stabilizing interaction later, but for now we simply model it phenomenologically. We will see that such timedependent mass squared terms with integer $n$ can arise naturally in plausible models.

Typically the mass scale of the potential will be very large compared with $H_{o}$. Thus following the phase transition $\phi$ will be coherently oscillating about the relatively slowly evolving time-dependent minimum of its potential. In order to discuss the time evolution of the $\phi$ energy density and equation of state, we follow the discussion of [14], based on averaging over the rapid oscillations of the scalar about the minimum of its potential.

The $\phi$ equation of motion is given by

$$
\ddot{\phi}+3 H \dot{\phi}=-\frac{\partial V}{\partial \phi} .
$$

This may be rewritten as

$$
\frac{\partial}{\partial t}\left(\frac{\dot{\phi}^{2}}{2}+V\right)=-3 H \dot{\phi}^{2},
$$

where the partial derivative is with respect to constant $\mu^{2}(t) .2$ Taking the time average over an oscillation cycle, we obtain

$$
\frac{\partial \rho_{\phi}}{\partial t}=-3 H \gamma \rho_{\phi}
$$

where

$$
\gamma=\frac{2 \int_{c}\left(1-V / V_{\max }\right)^{1 / 2} d \phi}{\int_{c}\left(1-V / V_{\max }\right)^{-1 / 2} d \phi} .
$$

$\int_{c}$ denotes integration over one oscillation cycle and $V_{\max } \equiv \rho_{\phi}$ is the maximum $\phi$ energy density during an oscillation cycle. $\gamma$ corresponds to the time average of $\dot{\phi}^{2} / \rho_{\phi}$

\footnotetext{
${ }^{2}$ In practice we evolve the energy density and pressure of $\phi$ by incrementing the energy density with $\mu(t)$ held constant and then incrementing $\mu(t)$ and calculating the pressure. Therefore we do not use the total derivative with respect to $t$ given in [14 for an explicitly time-dependent potential.
} 
over an oscillation cycle. The equation of state of the $\phi$ energy density is then given by

$$
\omega_{\phi} \equiv p_{\phi} / \rho_{\phi}=\gamma-1
$$

We have calculated the time evolution of the energy density and equation of state for different values of $n$ as a function of red-shift, $z$. The parameters of the potential are chosen such that we have at present $\Omega_{\phi} \approx 0.7$ and $\Omega_{m} \approx 0.3$ (where $\Omega_{m}$ is the density of conventional clustered dark matter).

The numerical solutions for $\rho_{\phi}$ and $\omega_{\phi}$ as a function of $z$ depend on $\mu_{o}^{2}$ and $\lambda$ only through the ratio $\mu_{o}^{4} / \lambda$; we have calculated the evolution for the case $\lambda=1$. Because of this scaling property, for a given value of $\omega_{\phi}$ today, $\omega_{\phi o}$, the evolution of the $\phi$ equation of state and energy density is completely fixed by the value of $n$ in the stabilizing interaction.

In Figure 1 we show the evolution of the equation of state $\omega_{\phi}$ as a function of red-shift for $n=2,3,4$. In Table 1 we give the parameters of the $\operatorname{VRPT}\left(\omega_{\phi o}, \mu_{o}\right.$ (for $\lambda=1$ ) and $z_{c}$, the red-shift at which the transition occurs) for the cases $n=2$ and $n=4$. For $n \geq 2$ and $\omega_{\phi o} \leq-0.6$ we find that the phase transition occurs at $z_{c} \leq 1.2$. (We consider $\omega_{\phi o} \leq-0.6$, in keeping with observational limits for the case of a fixed $\omega_{\phi}$ [15]. Since in our case $\omega_{\phi}$ is decreasing with $z$, this limit should be conservative.) From Table 1 we see that the mass of the scalar is $\lesssim 10^{-3} \mathrm{eV}$ for $\lambda \lesssim 1$. In Figure 2 we show the evolution of the energy density together with the matter energy density $\Omega_{m}$ for the cases $n=2$ and $n=4$, where we have normalized the energy density by taking the ratio to the present critical density $\rho_{c}$. Both $\omega_{\phi}$ and $\rho_{\phi}$ rapidly change at recent red-shifts, the more so for larger values of $n$.

In addition, we have considered the effect of the VRPT on the age of the Universe, given by

$$
t_{U}=\frac{2}{3} H_{o}^{-1} f_{U} \quad ; \quad f_{U}=\frac{3}{2} \int \frac{d a}{a}\left(\Omega_{\phi}(t)+\Omega_{m}(t)\right)^{-1 / 2}
$$

The age of globular clusters requires that $f_{U}=1.5 \pm 0.3$ [2, 11]. For a fixed cosmological constant and $\Omega_{\Lambda}=0.7, f_{U}=1.45$. For the VRPT $f_{U}$ is generally smaller, but not significantly so. The largest deviation in the examples considered corresponds to $n=2$ 

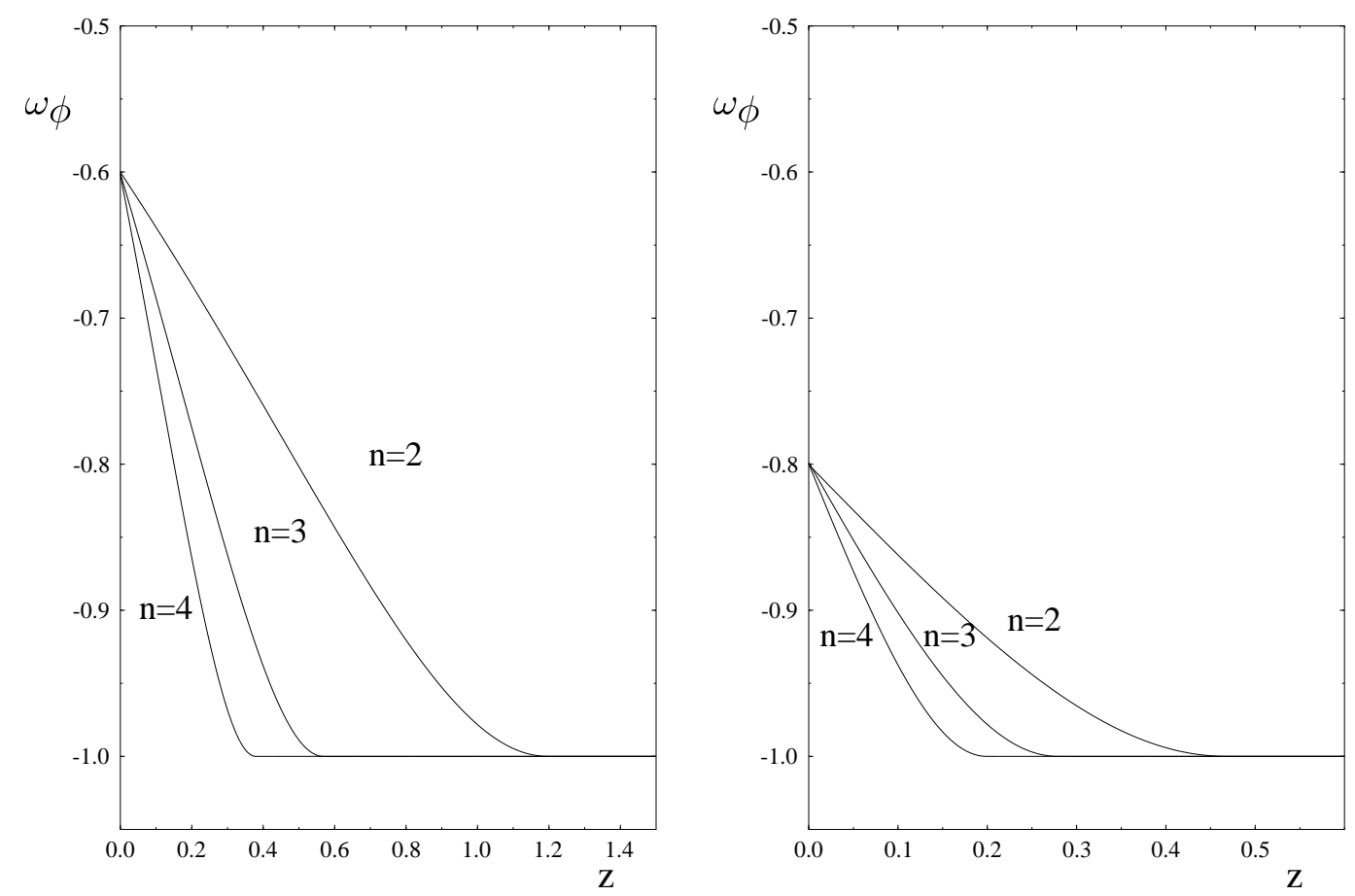

Figure 1: Evolution of the equation of state for different $n$ with $\omega_{\phi}=-0.6$ and $\omega_{\phi}=-0.8$ at present.

and $\omega_{\phi o}=-0.6$, for which $f_{U}=1.38$.

Table 1. VRPT parameters.

\begin{tabular}{|c|c|c|c|}
\hline $\mathrm{n}$ & $\omega_{\phi o}$ & $\mu_{o}$ & $z_{c}$ \\
\hline 2 & -0.6 & $3.45 \times 10^{-3} \mathrm{eV}$ & 1.20 \\
& -0.8 & $3.15 \times 10^{-3} \mathrm{eV}$ & 0.47 \\
\hline 3 & -0.6 & $3.27 \times 10^{-3} \mathrm{eV}$ & 0.57 \\
& -0.8 & $3.13 \times 10^{-3} \mathrm{eV}$ & 0.28 \\
\hline 4 & -0.6 & $3.21 \times 10^{-3} \mathrm{eV}$ & 0.38 \\
& -0.8 & $3.10 \times 10^{-3} \mathrm{eV}$ & 0.20 \\
\hline
\end{tabular}

After the phase transition has occured [3, the $\phi$ energy density will be composed of a time dependent vacuum energy $\rho_{\text {vac }}$ and a $\phi$ condensate component $\rho_{\text {osc }}$ corresponding to coherent oscillations about the time dependent minimum of the potential. In Figure

\footnotetext{
${ }^{3}$ We are assuming that $\phi$ is out of thermal equilibrium, so that the transition is purely dynamical in nature.
} 

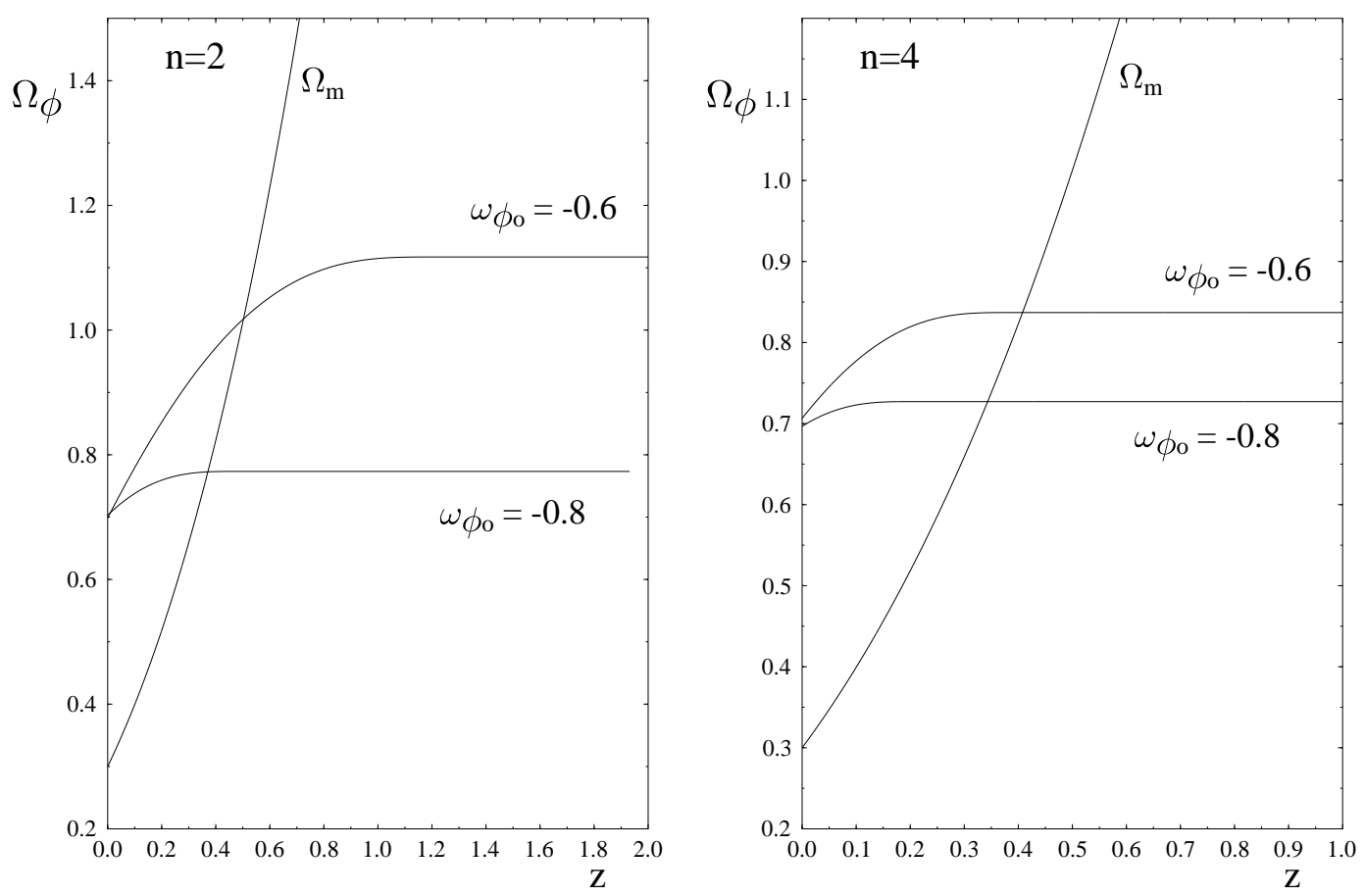

Figure 2: Evolution of the energy density $\Omega_{\phi}(t) \equiv \rho_{\phi}(t) / \rho_{c}$ for $n=2$ and $n=4$.

3 we show the equation of state and energy density of the $\phi$ oscillations as a function of $z$. We see that the equation of state rapidly tends towards the value $\omega_{\text {osc }}=0$ corresponding to effectively $\phi^{2}$ oscillations about the minimum. Nevertheless, the equation of state and so the pressure is significantly negative throughout. In Figure 4 we show the equation of state and energy density associated with the time dependent vacuum energy, $\rho_{v a c} \equiv V\left(\phi_{\min }(t)\right)$. We see that the equation of state does not significantly deviate from the value expected for a constant vacuum energy density, $\omega_{\Lambda}=-1$.

The negative pressure of the $\phi$ oscillations implies that the $\phi$ condensate is unstable with respect to spatial perturbations of $\phi$ 16, 17]. Such perturbations may be expected to exist, coming, for example, from thermal or inflationary quantum fluctuations. In the linear approximation the spatial perturbations evolve as

$$
\delta \ddot{\phi}_{\mathbf{k}}=\left|\omega_{\phi}\right| \mathbf{k}^{2} \delta \phi_{\mathbf{k}}
$$

(where $\omega_{\phi}<0$ is assumed). Therefore,

$$
\delta \phi_{\mathbf{k}}=\exp \left(\left|\omega_{\phi}\right|^{1 / 2}|\mathbf{k}| t\right) \delta \phi_{\mathbf{k} o}
$$



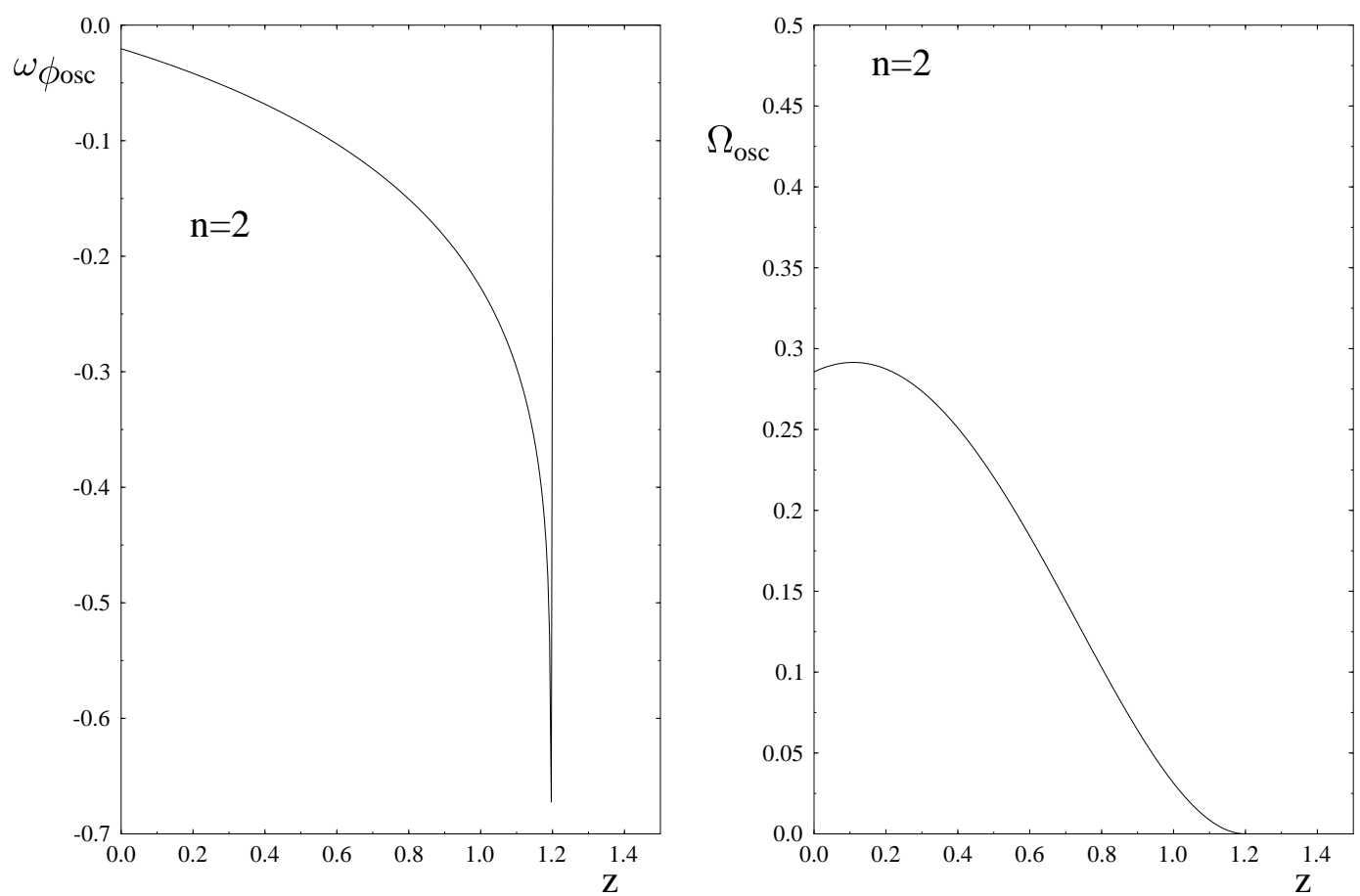

Figure 3: Evolution of the equation of state and energy density of the $\phi$ coherent oscillations.
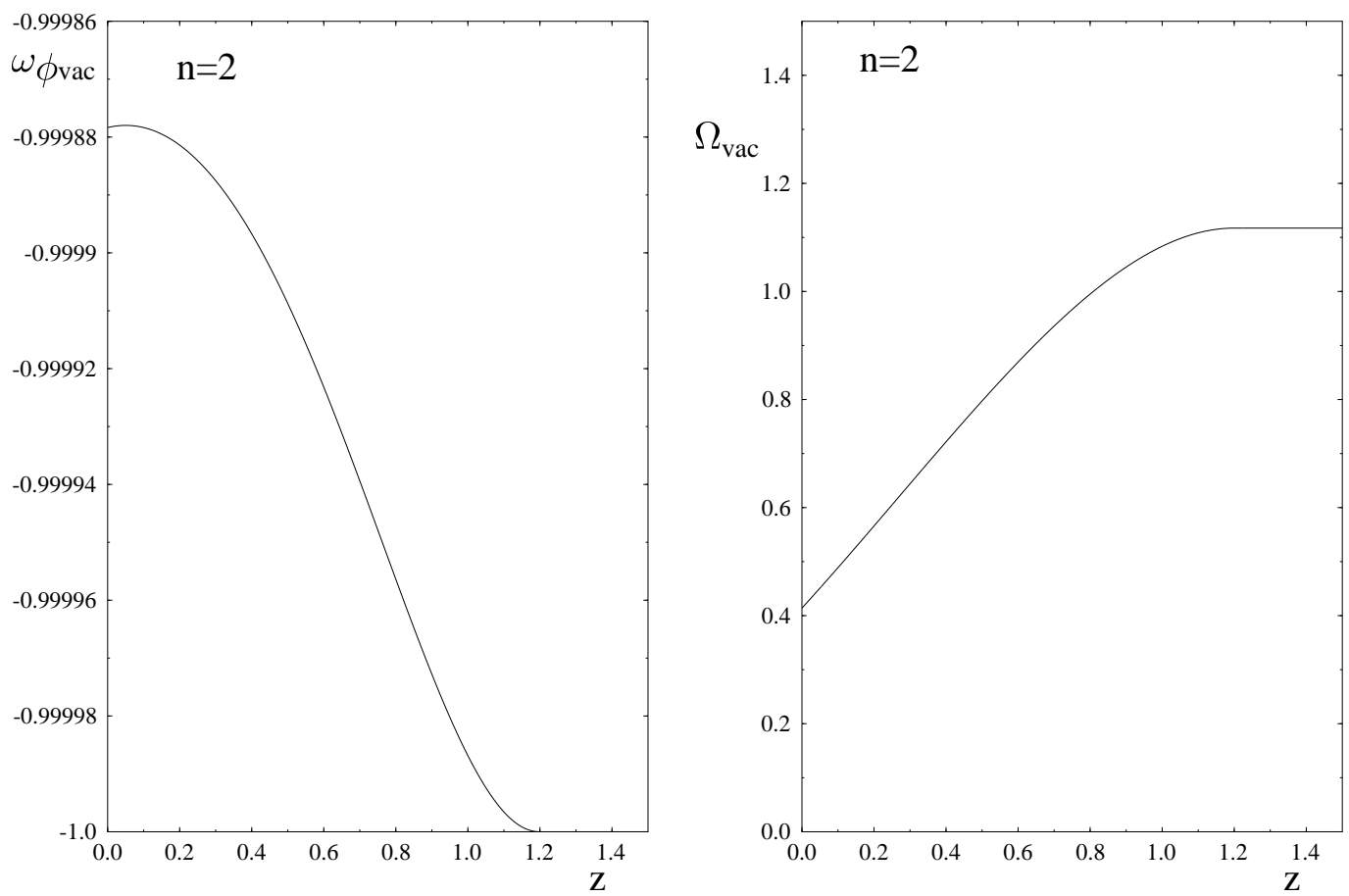

Figure 4: Evolution of the equation of state and energy density of the time dependent vacuum energy. 
This is true so long as the wavenumber of the perturbation satisfies $|\mathbf{k}|^{2} \approx 4\left|\omega_{\phi}\right| m_{\phi}^{2}$ [17], otherwise the positive pressure associated with gradient energy in the perturbation will overcome the negative pressure responsible for the growth of the perturbation. (For this reason, negative pressure effects play no role in the dynamics of $\Lambda(t)$ models based on slowly rolling fields, since their small mass, $m_{\phi}<H_{o}$, implies that perturbations on sub-horizon scales cannot grow.) The perturbations in the condensate will grow exponentially until they become non-linear. The condensate will then fragment into non-topological soliton lumps which we will refer to as ' $\phi$-axitons' (following the existence of similar objects in axion cosmology [18] and Affleck-Dine baryogenesis [19]). The radius of the $\phi$-axitons will be determined by the first perturbation mode to go non-linear, $r_{\phi} \approx\left(\left|\omega_{\phi}\right|^{1 / 2} m_{\phi}\right)^{-1}$ (assuming the exponential factor to be dominant in determining non-linearity). This will occur in a time $\delta t \approx\left(\left|\omega_{\phi}\right| m_{\phi}\right)^{-1} \log \left(\phi / \delta \phi_{\mathbf{k}}\right) \ll H_{o}^{-1}$.

Thus shortly after the VRPT the Universe will typically be filled with $\phi$-axitons. So long as $r_{\phi} \ll 10 \mathrm{Mpc}$, which is true if $m_{\phi} \gg 10^{-39} \mathrm{GeV}$, the $\phi$-axiton density will initially act as a smooth component of pressureless matter as far as determinations of the dark matter density are concerned.

We need to check that subsequent infall into galactic halos does not result in the $\phi$-axiton density clustering on 10Mpc scales and so no longer being effectively smooth. We do this via a simple Newtonian argument. We consider the mean distance between galaxies to be $R_{\text {gal }} \approx 10 \mathrm{Mpc}$, with $\phi$-axitons being smoothly distributed initially. The time scale for the $\phi$-axitons to fall a distance $\approx R_{\text {gal }}$ due to the attraction of a galaxy of mass $M_{g a l}$ is then

$$
t_{\text {infall }} \approx \frac{R_{\text {gal }}^{3 / 2}}{\sqrt{G M_{g a l}}}
$$

Using $M_{\text {gal }} \approx 10^{11} M_{\odot}$ for the mean galactic mass, this gives $t_{\text {infall }} \approx 1.6 \times 10^{12} \mathrm{yr}$, which is much longer than the age of the Universe, $t_{U} \approx 10^{10} \mathrm{yr}$. Thus infall will not significantly alter the smoothness of the $\phi$-axiton distribution. (The same argument holds for the $\phi$ particles in a homogeneous condensate.) Although we have estimated this at the present time, it holds for earlier times also, since $t_{U}\left(\propto H^{-1}\right)$ and $t_{\text {infall }}(\propto$ $R_{\text {gal }}^{3 / 2}$ ) are both proportional to $a^{3 / 2}$. 

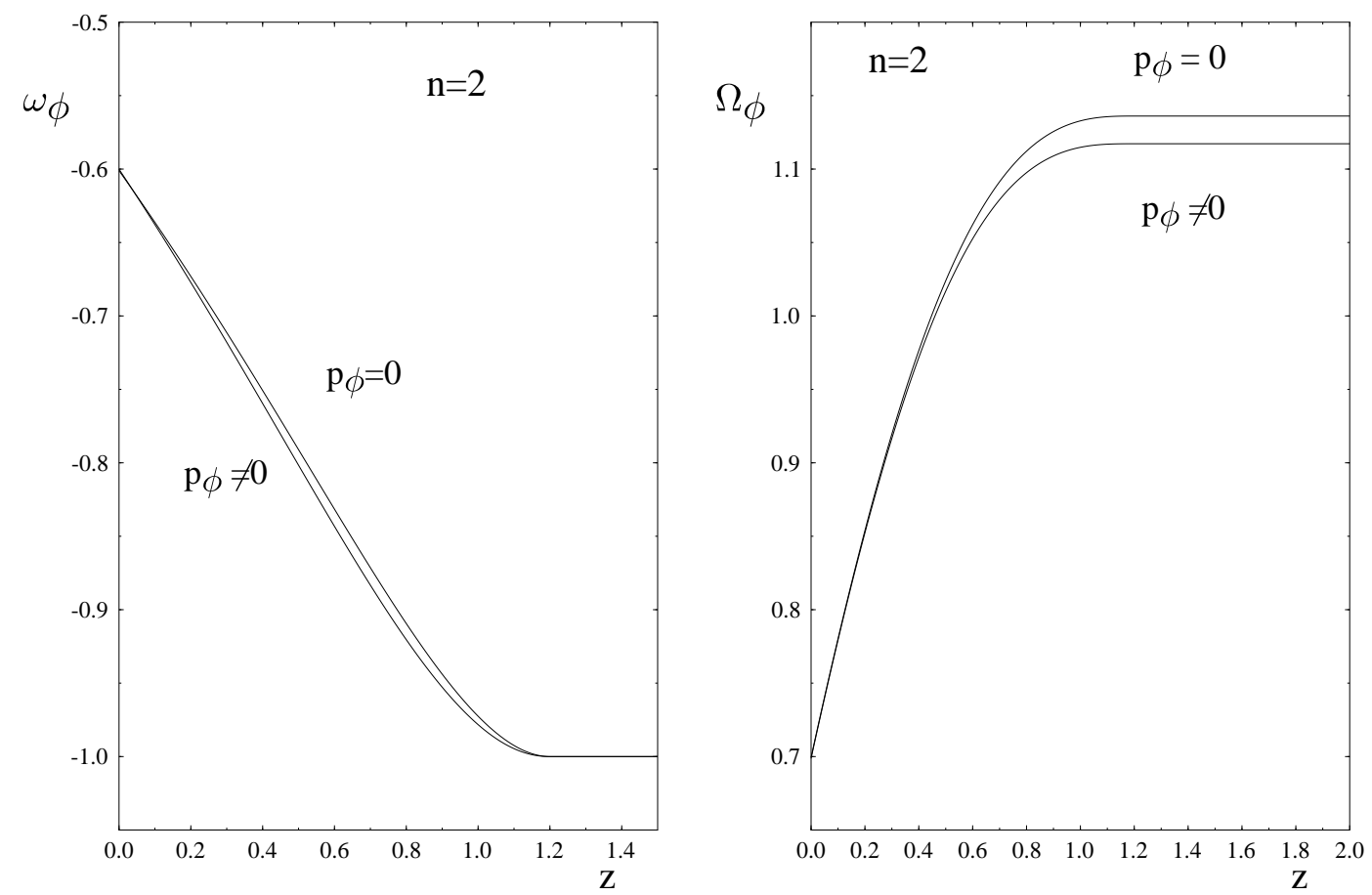

Figure 5: Evolution of the total equation of state and energy density for the case of pressureless $\phi$-axitons.

Therefore in the presence of spatial perturbations, the energy being fed into the coherent $\phi$ oscillations will be converted into a smooth density of pressureless $\phi$-axitons. This will slightly alter the evolution of the total $\phi$ equation of state and energy density from the case where there are coherent $\phi$ oscillations with negative pressure. In Figure 5 we show the effect of replacing the $\phi$ condensate with pressureless $\phi$-axiton matter. The effect is a small alteration of the total $\phi$ equation of state and energy density as a function of $z$.

After the phase transition, the $\phi$ energy density consists of a time-dependent vacuum energy

$$
\rho_{v a c}=\frac{\mu_{o}^{2}-\mu^{2}(t)}{4 \lambda}
$$

with $\omega_{v a c}=-1$ to a very good approximation, and an energy density either in the form of a coherently oscillating $\phi$ field with a negative pressure or, more likely, in the form of pressureless $\phi$-axitons. However, the total $\omega_{\phi}$ is insensitive to the pressure in the condensate component, as seen from Figure 5. This means that $\omega_{\phi}>-1$ following the VRPT is simply due to the dilution of $\omega_{\phi}$ by the low pressure component of smooth 
$\phi$ condensate or $\phi$-axiton matter,

$$
\omega_{\phi}=\frac{\omega_{o s c} \rho_{o s c}+\omega_{v a c} \rho_{v a c}}{\rho_{o s c}+\rho_{v a c}} \approx \frac{-\rho_{v a c}}{\rho_{o s c}+\rho_{v a c}} .
$$

So far we have introduced a simple time-dependent mass squared term ('stabilizing interaction') in order to trigger the phase transition and so produce a dynamical cosmological constant. We now consider some ways in which this term could be generated in particle physics models. One possibility is that the $\phi$ field might couple to a light field which has a thermal distribution, such as the photons or neutrinos. For example, one could consider a coupling of the form $\frac{\phi^{2}}{M^{2}} \mathcal{L}_{k e}$, where $\mathcal{L}_{k e}=-\frac{1}{4} F^{\mu \nu} F_{\mu \nu}, \bar{\psi} \partial_{\mu} \gamma^{\mu} \psi$. On taking the average over thermal fluctuations of the fields in $\mathcal{L}_{k e}$ we obtain an effective $\phi^{2}$ term $\sim \frac{T^{4}}{M^{2}} \phi^{2}$. With $T \propto a^{-1}$ this results in a stabilizing interaction with $n=4$. Alternatively we might consider a very light additional scalar field $\chi$ with a thermal distribution (and a small enough energy density so as to avoid nucleosynthesis constraints), coupling to $\phi$ via a term $\chi^{2} \phi^{2}$. On averaging over thermal fluctuations of the $\chi$ field, this will give an effective $\phi^{2}$ term $\sim T^{2} \phi^{2}$, corresponding to a stabilizing interaction with $n=2$. In both of these interactions we have effectively massless fields coupling to $\phi$, so that the time dependence of the stablilzing interaction is due to the red-shifting of the energy of the thermal fluctuations of the light fields. An alternative is to consider the case where $\chi$ is massive and is coherently oscillating in an effectively $\chi^{2}$ potential about $\chi=0$. We assume that the $\chi$-matter density is negligible compared with the conventional dark matter density. In this case the $\chi$ oscillation amplitude will be proportional to $a^{-3 / 2}$, resulting in a stabilizing interaction with $n=3$. These are just a few examples; one could also consider, for example, $\chi$ to be a slowly rolling field which triggers the phase transition, analogous to what happens in hybrid inflation models [20].

In conclusion, we have introduced an alternative model for a dynamical cosmological constant, based on the idea that a scalar field underwent a phase transition from a metastable phase at a very recent epoch, $z \leq 1.2$. This VRPT scenario results in a characteristic evolution of the equation of state and energy density which is quite distinct from the case of models based on slowly rolling fields, with the pressure rapidly rising from $\omega_{\phi}=-1$ and $\rho_{\phi}$ rapidly decreasing at very recent times. The solutions for 
$\omega_{\phi}(z)$ and $\rho_{\phi}(z)$ are uniquely determined by the present value of $\omega_{\phi}$ and the form of the time dependent mass squared term in the potential. Following the phase transition, the $\phi$ energy density will consist of a time dependent vacuum energy and a negative pressure $\phi$ condensate which typically fragments to a smooth pressureless density of non-topological solitons, ' $\phi$-axitons'.

As with most other dynamical $\Lambda$ models, it is implicitly assumed that the reason for the recent dominance of the $\phi$ energy density and the recent occurance of the phase transition is connected with anthropic selection. In general, dynamical $\Lambda$ models require two conditions; that the energy density in the scalar field has recently become dominant and that the scalar field energy density is varying significantly on time scales of the order of $H_{o}^{-1}$. Therefore two tunings are generally required. In this regard the VRPT scenario is on the same footing as other dynamical $\Lambda$ models. Ultimately the question of which dynamical $\Lambda$ model is correct is a matter to be decided by observations. A future goal will therefore be to understand the detailed predictions of this class of dynamical $\Lambda$ model for observable quantities; high-z supernova, quasar lensing statistics, the galaxy clustering power spectrum and in particular the angular CMB spectrum. We expect that these will be clearly distinguishable from other dynamical $\Lambda$ models. In addition, the idea of a very recent phase transition leads to other interesting issues. One is whether the $\phi$-axiton density could be observationally or experimentally distinguished from conventional cold dark matter. This will depend on the $\phi$ mass and on how strongly the $\phi$ field interacts with ordinary matter. In addition, it is possible that the VRPT could result in the very recent formation of domain walls or global strings, depending on the symmetry associated with the potential and whether $\phi$ is real or complex. It would be interesting to consider whether there are any observable effects associated with the very recent creation of topological defects. With the possibility of recently formed topological and non-topological solitons, the phenomenology of the VRPT scenario for the dynamical cosmological constant may be surprisingly rich. 


\section{References}

[1] A.J.Riess et al., Astron.J. 116 (1998) 1009; S.Perlmutter et al., Ap. J. 517 (1999) 565; N.Bachall, J-P.Ostriker, S.Perlmutter and P.J.Steinhardt, Science 284 (1999) 1481.

[2] L.Krauss and M.S.Turner, Gen. Rel. Grav. 27 (1995) 1137; J-P.Ostriker and P.J.Steinhardt, Nature 377 (1995) 600.

[3] S.Dodelson and L.Knox, Phys. Rev. Lett. 84 (2000) 3523 ; A.Melchiorri et al., Ap. J. 536 (2000) L63; M.Tegmark and M.Zaldarriaga, astro-ph/0002091; G.Efstathiou, astro-ph/0002249.

[4] S.Weinberg, Talk at DM2000, Marina del Ray, CA, USA. 23-25 Feb. 2000. astroph/0005265

[5] C.Wetterich, Astron. and Astrophys. 301 (1995) 321; P.G.Ferreira and M.Joyce, Phys. Rev. D58 (1998) 023503.

[6] S.Dodelson, M.Kaplinghat and E.Stewart, astro-ph/0002360.

[7] C.Armendariz-Picon, V.Mukhanov and P.J.Steinhardt, astro-ph/0004134.

[8] J.A.Frieman and I.Waga, Phys. Rev. D57 (1998) 4642.

[9] I.Waga and J.A.Frieman, astro-ph/0001354.

[10] J.A.Frieman, C.T.Hill, A.Stebbins and I.Waga, Phys. Rev. Lett. 75 (1995) 2077.

[11] K.Coble, S.Dodelson and J.A.Frieman, Phys. Rev. D55 (1997) 1851.

[12] R.R.Caldwell, R.Dave and P.J.Steinhardt, Phys. Rev. Lett. 80 (1998) 1582.

[13] P.J.E.Peebles and B.Ratra, Ap. J. 325 (1988) L17; B.Ratra and P.J.E.Peebles, Phys. Rev. D37 (1988) 3406; I.Zlatev, L.Wang and P.J.Steinhardt, Phys. Rev. Lett. 82 (1998) 896; P.J.Steinhardt, L.Wang and I.Zlatev, Phys. Rev. D59 (1999) 123504. 
[14] M.S.Turner, Phys. Rev. D28 (1983) 1243.

[15] L.Wang, R.R.Caldwell, J.P.Ostriker and P.J.Steinhardt, Ap. J. 530 (2000) 17.

[16] J.McDonald, Phys. Rev. D48 (1993) 2573.

[17] A.Kusenko and M.Shaposhnikov, Phys. Lett. B418 (1998) 46; K.Enqvist and J.McDonald, Phys. Lett. B425 (1998) 309; Nucl. Phys. B538 (1999) 321.

[18] E.Kolb and I.Tkachev, Phys. Rev. D49 (1994) 5040.

[19] K.Enqvist and J.McDonald, Nucl. Phys. B570 (2000) 407.

[20] K.Enqvist and D.V.Nanopoulos, Phys. Lett. B142 (1984) 349; A.D.Linde, Phys. Rev. D49 (1994) 748. 